\title{
Polarized Fermi Condensates with Unequal Masses: Tuning the Tricritical Point
}

\author{
M. M. Parish, ${ }^{1, *}$ F. M. Marchetti, ${ }^{1, \dagger}$ A. Lamacraft, ${ }^{2}$ and B. D. Simons ${ }^{1}$ \\ ${ }^{1}$ Cavendish Laboratory, J. J. Thomson Avenue, Cambridge, CB3 OHE, United Kingdom \\ ${ }^{2}$ Rudolf Peierls Centre for Theoretical Physics, 1 Keble Road, Oxford OX1 3NP, United Kingdom
}

(Received 31 August 2006; published 18 April 2007)

\begin{abstract}
We consider a two-component atomic Fermi gas within a mean-field, single-channel model, where both the mass and population of each component are unequal. We show that the tricritical point at zero temperature evolves smoothly from the BEC to BCS side of the resonance as a function of mass ratio $r$. We find that the interior gap state proposed by Liu and Wilczek is always unstable to phase separation, while the breached pair state with one Fermi surface for the excess fermions exhibits differences in its density of states and pair correlation functions depending on which side of the resonance it lies. Finally, we show that, when $r \gtrsim 3.95$, the finite-temperature phase diagram of trapped gases at unitarity becomes topologically distinct from the equal mass system.
\end{abstract}

DOI: 10.1103/PhysRevLett.98.160402

Recent advances in the ability to manipulate and control ultracold atomic vapors have provided a unique experimental system in which to explore pairing phenomena. Following the successful realization of the crossover from the BCS to the Bose-Einstein condensed phase [1], attention has turned to the consideration of more exotic Fermi superfluids. A subject that has attracted particular interest is that of Fermi condensates with imbalanced spin populations [2,3], having relevance to QCD and magnetized superconductors [4]. Equally intriguing is the case in which both the mass and population of each fermionic species in the condensate are unequal. Indeed, the realization of Feshbach resonances in Fermi-Bose mixtures [5] and the predicted stability of diatomic molecules close to resonance over a wide range of mass ratios [6] suggest that such superfluid mixtures should be experimentally accessible.

Previous studies [7-10] have raised several important issues unique to Fermi condensates with spin and mass imbalances. First, it has been proposed that the breached pair (BP) state, where the superfluid and excess fermionic states phase separate in momentum space, can possess excess fermions with two Fermi surfaces (BP-2) - the interior gap state [7]. In the case of equal masses, the BP state can only have one Fermi surface (BP-1). However, it is still unclear whether BP-2 can become stable for large mass ratios near the Feshbach resonance [11], or whether it is always unstable to phase separation in real space as in the weak-coupling limit [12]. Second, although there have been studies of the zero temperature phase diagram for the homogeneous gas with unequal masses [8,9], so far no tricritical point (such as that discussed in the equal mass system $[13,14]$ ) has been identified. It is natural to ask whether such a tricritical point is generic and, if so, how it evolves with mass ratio. Finally, it has been shown that trapped Fermi gases with unequal masses can exhibit spatial phase separation at zero temperature that differs qualitatively from that of the equal mass case [10]. Whether and how such features extend to finite tempera-
PACS numbers: 03.75.Hh, 03.75.Ss, 05.30.Fk, 74.20.-z

tures remains unanswered. Focusing on the homogeneous system, all three issues will be addressed in this work. To focus our discussion, we will address the phase boundaries between spatially homogeneous phases, leaving the potential for Fulde-Ferrell-Larkin-Ovchinnikov phases [15] to future investigation.

Referring to wide (viz. entrance-channel dominated) Feshbach resonances [16], we restrict attention to a single-channel Hamiltonian with a contact potential:

$$
\begin{aligned}
\hat{H}-\sum_{\sigma=\uparrow, \downarrow} \mu_{\sigma} \hat{n}_{\sigma}= & \sum_{\mathbf{k} \sigma}\left(\epsilon_{\mathbf{k} \sigma}-\mu_{\sigma}\right) c_{\mathbf{k} \sigma}^{\dagger} c_{\mathbf{k} \sigma}+\frac{g}{V} \\
& \times \sum_{\mathbf{k}, \mathbf{k}^{\prime}, \mathbf{q}} c_{\mathbf{k}+\mathbf{q} / 2 \uparrow}^{\dagger} c_{-\mathbf{k}+\mathbf{q} / 2 \downarrow}^{\dagger} c_{-\mathbf{k}^{\prime}+\mathbf{q} / 2 \downarrow} c_{\mathbf{k}^{\prime}+\mathbf{q} / 2 \uparrow}
\end{aligned}
$$

Here, $\epsilon_{\mathbf{k} \sigma}=\frac{\mathbf{k}^{2}}{2 m_{\sigma}}(\hbar=1), g$ is the interaction strength, $V$ is the volume, and we allow the mass $m_{\sigma}$ and chemical potential $\mu_{\sigma}$ of each spin to be different, with average chemical potential $\mu=\left(\mu_{\uparrow}+\mu_{\downarrow}\right) / 2$ and "Zeeman" field $h=\left(\mu_{\uparrow}-\mu_{\downarrow}\right) / 2$. To obtain the topology of the phase diagram spanning the BCS-BEC limits for unequal masses (including the locus of the finite-temperature tricritical point at which the transition between the superfluid and normal phases switches from first to second order), we will develop a mean-field analysis of the system analogous to that presented for equal masses [13]. While such a treatment is not expected to be quantitatively correct for all interaction strengths, it should provide a reliable qualitative description even close to unitarity.

Setting $\epsilon_{\mathbf{k}+}=\frac{\mathbf{k}^{2}}{2 m_{r}}, \frac{2}{m_{r}}=\frac{1}{m_{\uparrow}}+\frac{1}{m_{\downarrow}}, \epsilon_{\mathbf{k}-}=\epsilon_{\mathbf{k}+} \frac{(r-1)}{(r+1)}$, and $r=\frac{m_{\downarrow}}{m_{\mathrm{\downarrow}}}$, the free energy density can be expressed as

$$
\begin{aligned}
\Omega^{0}(\mu, h)= & \min _{\Delta}\left\{-\frac{\Delta^{2}}{g}\right. \\
& \left.+\frac{1}{V} \sum_{\mathbf{k}}\left[\xi_{\mathbf{k}}-E_{\mathbf{k}}-\frac{1}{\beta} \sum_{\sigma} \ln \left(1+e^{-\beta E_{\mathbf{k} \sigma}}\right)\right]\right\},
\end{aligned}
$$


where $\min _{\Delta}$ gives the global minimum with respect to $\Delta$ (to be calculated numerically). Here, $\xi_{\mathbf{k}}=\epsilon_{\mathbf{k}+}-\mu$ and, defining $E_{\mathbf{k}}=\sqrt{\xi_{\mathbf{k}}^{2}+\Delta^{2}}$, the quasiparticle energies are given by $E_{\mathbf{k} \sigma}=E_{\mathbf{k}} \mp\left(h-\epsilon_{\mathbf{k}-}\right)$. The free energy (2) differs from that of the equal mass system only through the appearance of a momentum-dependent contribution, $\epsilon_{\mathrm{k}-}$, to the Zeeman term which leads to the symmetry $(h, r) \mapsto(-h, 1 / r)$. We introduce the $s$-wave scattering length $a$ via the prescription $\frac{m_{r} V}{4 \pi a}=\frac{V}{g}+\sum_{\mathbf{k}}^{k_{0}} \frac{1}{2 \epsilon_{\mathbf{k}+}}$, where the UV cutoff $k_{0}$ can be sent to infinity at the end of the calculation. With experiments performed at fixed density, $\mu$ and $h$ are determined from the total density $n \equiv n_{\uparrow}+$ $n_{\downarrow}=-\partial \Omega^{0} / \partial \mu$ and the population imbalance $m \equiv n_{\uparrow}-$ $n_{\downarrow}=-\partial \Omega^{0} / \partial h$ [17]. Note that the stability criterion adopted in Refs. [8,9] corresponds only to finding local minima with respect to $\Delta$ in Eq. (2) and thus will not correctly locate a first-order transition. The presence of such a transition implies a region of phase separation in real space if $n$ and $m$ are held fixed [12]. The two phases in question are a normal phase with $\Delta=0$ and a superfluid phase with $\Delta \neq 0$, which is less magnetized as pairing tends to enforce equality of populations.

Beginning with zero temperature (Fig. 1), we find that the basic structure of the phase diagram $\left(\frac{1}{k_{F} a}, \frac{m}{n}\right)$ for unequal masses mirrors that of the equal mass system [18]. Phase separation (PS) is found between the normal (N) and superfluid states. On the BCS side the superfluid component is always unpolarized, while the BP or magnetized superfluid $\left(\mathrm{SF}_{\mathrm{M}}\right)$ state exists for sufficiently strong interaction and eventually undergoes a second-order transition to the fully polarized normal state beyond the tricritical point. However, the position of the tricritical point is dramatically shifted, with the regions of $\mathrm{PS}$ and $\mathrm{SF}_{\mathrm{M}}$ shrinking and expanding, respectively, as $r$ increases (in qualitative agreement with Refs. [8-10]).

We can gain further insight into the phase diagram by examining the behavior of the tricritical point as a function of mass ratio, $r$. As shown in Fig. 2, the tricritical point, determined as the point at which both the quadratic and quartic terms in the Landau expansion of the free energy (2) vanish, evolves smoothly from the BEC to BCS limits, with $\left(h / \varepsilon_{F}, 1 / k_{F} a\right)_{\text {tric }} \rightarrow(\infty, \infty)$ as $r \rightarrow 0$ and $\left(h / \varepsilon_{F}, 1 / k_{F} a\right)_{\text {tric }} \rightarrow\left(2^{2 / 3},-\infty\right)$ as $r \rightarrow \infty$. Moreover, as in the equal mass system, the tricritical point always corresponds to a fully polarized state, $m / n=1$ [19]. It is interesting to note (Fig. 2) that the chemical potential, $\mu$, at the tricritical point becomes positive for $r \geq 3$, hinting at the possibility of a BP-2 state. However, a mean-field analysis of the phase boundaries in the limit $r \rightarrow \infty$ shows that only the BP-1 state is ever stable. As in the weakcoupling limit [12], phase separation is always favored over the BP-2 state [20].

Even though the BP state only ever possesses one Fermi surface, the associated quasiparticle excitation spectrum can display minima at nonzero $\mathbf{k}$, a property which is also

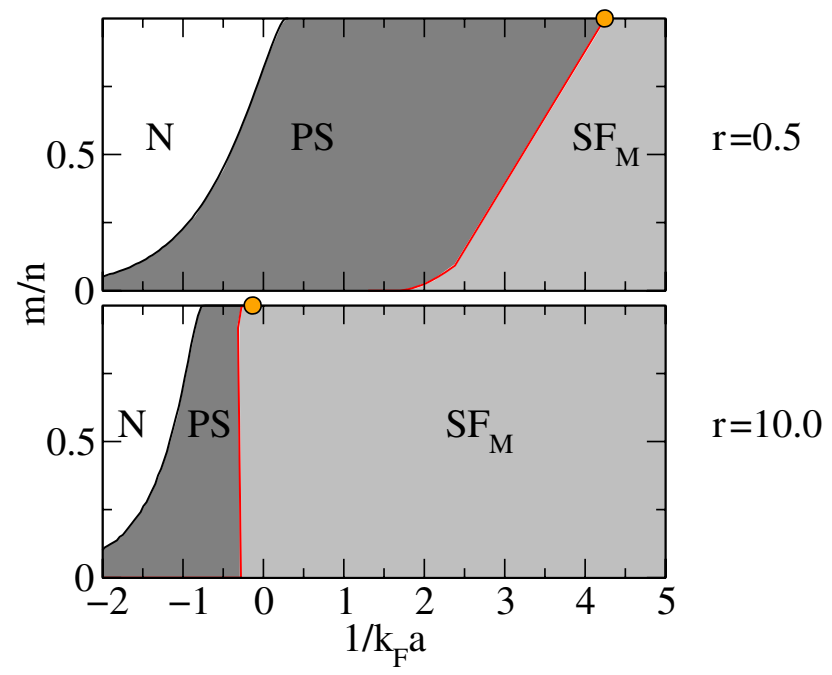

FIG. 1 (color online). Zero temperature phase diagrams for different mass ratios $r=m_{\downarrow} / m_{\uparrow}$, where a positive polarization $m$ corresponds to an excess of particles with spin $\uparrow$. All mass ratios exhibit the same three phases: the normal state $(\mathrm{N})$, the spatially homogeneous superfluid $\left(\mathrm{SF}_{\mathrm{M}}\right)$, and phase separation (PS) between normal and superfluid states in real space. The region of $\mathrm{SF}_{\mathrm{M}}$ expands with increasing $r$. The boundaries enclosing the PS region are all first order, while the tricritical point is represented by a circle. The line defined by $m / n=0$ corresponds to the usual BCS-BEC crossover.

characteristic of the BP-2 phase. As demonstrated in Fig. 3, while the spectrum remains gapless for both $r=0.5$ and $r=10$ [i.e., the density of states, $\operatorname{DOS}(\omega)$, is finite at $\omega=$ $0]$, the excitation spectrum for $r=10$ also exhibits stationary points at nonzero $\mathbf{k}$ resulting in a square-root singularity in the associated DOS. Such a singularity may be regarded as "BCS-like," while its absence for $r=$ 0.5 is "BEC-like" (cf. quasiparticle excitation spectra at the BCS-BEC crossover in the equal mass case [21]). More generally, DOS singularities in the BP state only occur for $r>1$, where the species with the smaller mass is in the majority. When $\mu>0$, the singularities exist for all polarizations, but when $\mu<0$, they are restricted to the

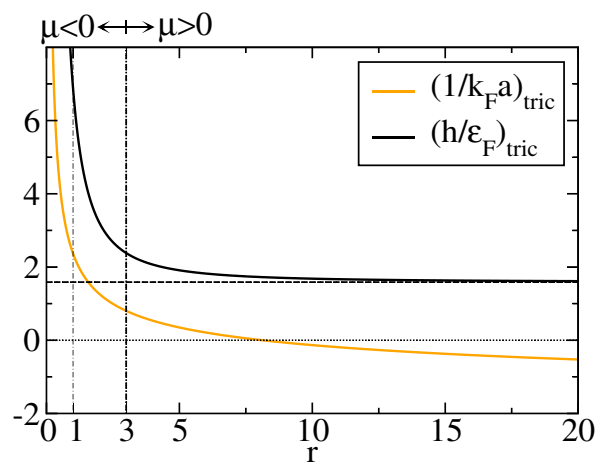

FIG. 2 (color online). Evolution of the tricritical point $\left(1 / k_{F} a, h / \varepsilon_{F}\right)_{\text {tric }}$ as a function of mass ratio $r$ with $h>0$. The values of $r=1$, the equal mass case, and $r=3$, where the chemical potential $\mu$ changes sign, are explicitly marked. 
region around $m=0$ where $\Delta /|\mu|>2 \sqrt{r} /(r-1)$ [22]. Such dramatic differences in the DOS should be accessible experimentally using optical excitations [23].

Further signatures of breached pairing are visible in the momentum distribution, $n_{\mathbf{k} \sigma}$, and correlation function,

$$
\begin{aligned}
C_{\uparrow \downarrow}\left(\mathbf{k}_{1}, \mathbf{k}_{2}\right) & =\left\langle\hat{n}_{\mathbf{k}_{1} \uparrow} \hat{n}_{\mathbf{k}_{2} \downarrow}\right\rangle-\left\langle\hat{n}_{\mathbf{k}_{1} \uparrow}\right\rangle\left\langle\hat{n}_{\mathbf{k}_{2} \downarrow}\right\rangle \\
& =\delta_{\mathbf{k}_{1},-\mathbf{k}_{2}} \frac{\Delta^{2}}{4 E_{\mathbf{k}_{1}}^{2}}\left[1-\Theta\left(-E_{\mathbf{k} \uparrow}\right)-\Theta\left(-E_{\mathbf{k} \downarrow}\right)\right]^{2} .
\end{aligned}
$$

Referring to Fig. 4, breached pairing is characterized by a phase separation in momentum space between the excess of majority species $\uparrow$ and the minority species $\downarrow$ involved in the superfluid state. In both cases, the correlation function $C_{\uparrow \downarrow}$ shows a "hole" for momenta less than the Fermi momentum of the majority quasiparticles. This hole at small momenta is reminiscent of the Pauli blocking observed in the closed-channel molecule of the ${ }^{40} \mathrm{~K}$ Feshbach resonance [24] where there is always an inherent particle number asymmetry in the open channel, even in the usual BCS-BEC crossover. The correlation function $C_{\uparrow \downarrow}$ can also directly probe the sign of $\mu$ : for $\mu>0$ we have a peak beyond the blocked region, as shown in the $r=10.0$ case, provided $\Delta / \mu>|h / \mu+(1-r) /(1+r)|$. In principle, such a feature can be detected experimentally using noise correlations [25].

Turning to finite temperature, the phase diagram for unequal masses in the homogenous system is qualitatively similar to the equal mass case, but the topology of phases within a trap can be different. A study of ${ }^{6} \mathrm{Li}-{ }^{40} \mathrm{~K}$ mixtures at zero temperature [10] has already revealed that the unequal mass case offers a richer variety of phaseseparated states. In addition to configurations where there is a superfluid core surrounded by the normal state (as in

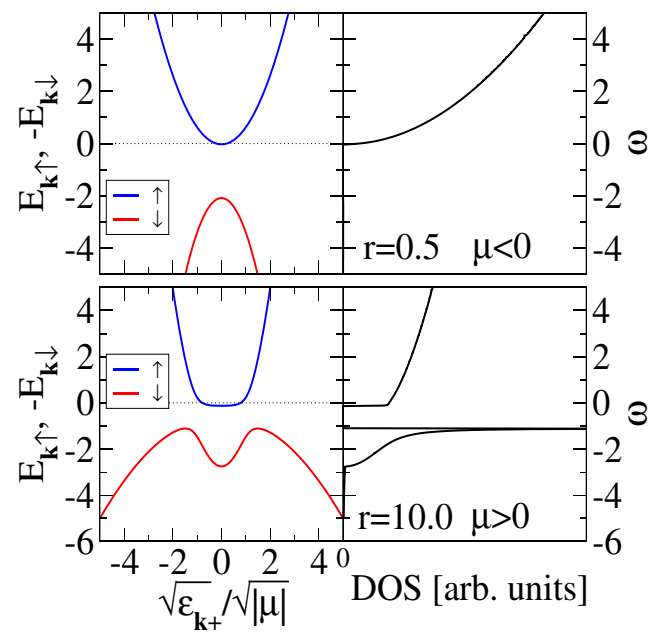

FIG. 3 (color online). Quasiparticle dispersions $E_{\mathbf{k} \uparrow}, E_{\mathbf{k} \downarrow}$ together with the majority species ( $\uparrow$ ) projection of the $\operatorname{DOS}(\omega)=-\frac{1}{\pi} \sum_{\mathbf{k}} \Im\left[G_{11}\left(\mathbf{k}, i \epsilon_{n}=\omega+i \eta\right)\right]$ for mass ratios $r=0.5$ (upper panels) $\left(\Delta /|\mu|=0.25, h /|\mu|=1.06,1 / k_{F} a=\right.$ 2.93, $m / n=0.12$ ) and $r=10.0$ (lower panels) $(\Delta / \mu=0.85$, $\left.h /|\mu|=1.44,1 / k_{F} a=-0.07, m / n=0.16\right)$. the equal mass case), the superfluid region can occupy a shell sandwiched between a normal inner core and a normal outer shell with opposite polarizations. To investigate the effects of temperature $T$ and different mass ratios $r$ in the trap geometry, we restrict ourselves to the case in which the local density approximation can be applied. Thus, assuming each species experiences the same trapping potential $V(\mathbf{r})$, the effects of the trap can be included in the spatially varying chemical potential, $\mu-V(\mathbf{r})$, while the term $h$ remains fixed.

In Fig. 5, we plot mean-field phase diagrams at unitarity as a function of $T / \mu$ and $h / \mu$. Note that NozièresSchmitt-Rink fluctuations will not alter these diagrams since corrections only enter into the density, $n$, and the polarization, $m$ [26]. We restrict ourselves to the case where $\mu>0$ because this is enough to completely encompass the SF-N phase boundaries, even though a fully polarized normal state obviously exists for $-|h|<\mu<$ 0 . While the equal mass $(r=1)$ phase diagram is symmetric around $h=0$ as expected, we see that lowering or increasing $r$ translates the superfluid dome to the left or right, respectively. For sufficiently small translations, we have the ordinary trapped case of a superfluid core with a first (arrow $a$ ) or second (arrow $b$ ) order transition to the surrounding normal state. However, once $r \gtrless 3.95$, the superfluid region has shifted entirely to the $h>0$ plane, as shown for $r=6.7$ and $r=10$ [27]. Provided $h / \mu$ is sufficiently small at the trap center, this naturally leads to a superfluid shell structure where the superfluid phase is sandwiched between a "heavy" normal core and an outer "light" normal phase. This structure can either have two first-order SF-N phase boundaries $(d)$, one first and one second $(c)$, or two second-order phase boundaries $(e)$, depending on the value of $T / h$. The case considered in Ref. [10] corresponds to the $T / \mu=0$ axis in the $r=6.7$ phase diagram, and is thus an example of category $d$. Note, however, that one tricritical point vanishes when $r$ is further increased [corresponding to the point where

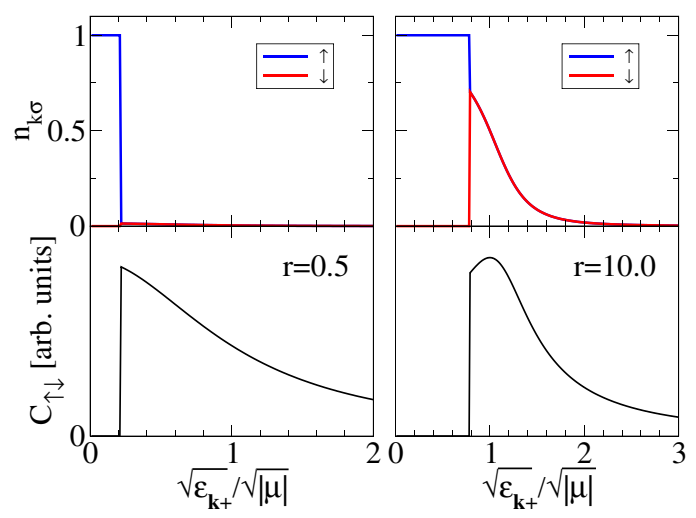

FIG. 4 (color online). Averaged momentum distribution for each spin state $n_{\mathbf{k} \uparrow, \downarrow}$ and correlation function $C_{\uparrow \downarrow}$ for $r=0.5$ (left panels) and $r=10.0$ (right panels) and the same parameter values used in Fig. 3. 


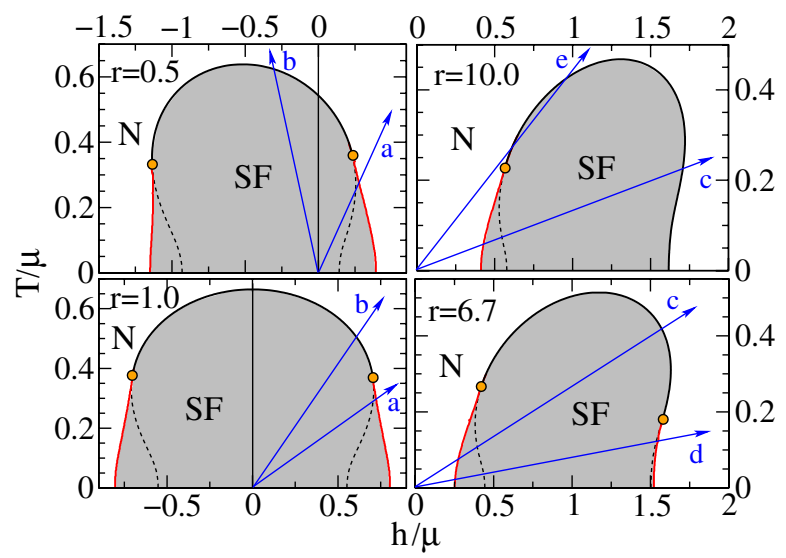

FIG. 5 (color online). Phase diagrams in the $(T / \mu, h / \mu)$ plane for different mass ratios $r$ at unitarity $1 / k_{F} a=0$. Mass ratio $r=$ 6.7 corresponds to the case of ${ }^{6} \mathrm{Li}^{-40} \mathrm{~K}$ mixtures. The transition between the $\mathrm{N}$ and SF phase can be first (red line) or second order (black solid line), separated by the tricritical point. The dashed black line describes the second-order transition in the region where the true transition is first order. Lines of constant gradient $T / h$ represent the trajectory traveled from the center to the edges of the trap, examples of which are shown by the arrows (a) $-(\mathrm{e})$. The starting points on these lines are the values of $(T / \mu$, $h / \mu)$ at the trap center.

$\left(1 / k_{F} a\right)_{\text {tric }}$ changes sign in Fig. 2], so that "d-type" SF shells are then no longer possible.

In conclusion, we have investigated the mean-field phase diagram of a polarized Fermi condensate with unequal masses. We have shown that the zero temperature tricritical point smoothly crosses over from $1 / k_{F} a>0$ to $1 / k_{F} a<0$ as $r$ increases, but the interior gap state is never stable, even in the limit of infinite $r$. However, differences in the BP states do show up in the DOS and pair correlations. Finally, we show how the phase diagram of trapped gases depends on $r$ and $T$, including how one obtains superfluid shells for $r \gtrsim 3.95$.

*Electronic address: mmp24@cam.ac.uk †Electronic address: fmm25@cam.ac.uk

[1] C. A. Regal et al., Phys. Rev. Lett. 92, 040403 (2004); M. W. Zwierlein et al., Phys. Rev. Lett. 92, 120403 (2004); C. Chin et al., Science 305, 1128 (2004); T. Bourdel et al., Phys. Rev. Lett. 93, 050401 (2004); J. Kinast et al., Phys. Rev. Lett. 92, 150402 (2004); M. Zwierlein et al., Nature (London) 435, 1047 (2005).

[2] See, e.g., P. F. Bedaque et al., Phys. Rev. Lett. 91, 247002 (2003); J. Carlson and S. Reddy, Phys. Rev. Lett. 95, 060401 (2005); C.-H. Pao et al., Phys. Rev. B 73, 132506 (2006); D. T. Son and M. A. Stephanov, Phys.
Rev. A 74, 013614 (2006); T. Mizushima et al., Phys. Rev. Lett. 94, 060404 (2005); D. E. Sheehy and L. Radzihovsky, Phys. Rev. Lett. 96, 060401 (2006).

[3] M.W. Zwierlein et al., Science 311, 492 (2006); G. B. Partridge et al., Science 311, 503 (2006); M. W. Zwierlein et al., Nature (London) 442, 54 (2006); Y. Shin et al., Phys. Rev. Lett. 97, 030401 (2006).

[4] R. Casalbuoni and G. Nardulli, Rev. Mod. Phys. 76, 263 (2004).

[5] C. A. Stan et al., Phys. Rev. Lett. 93, 143001 (2004); S. Inouye et al., Phys. Rev. Lett. 93, 183201 (2004).

[6] D. Petrov et al., J. Phys. B 38, S645 (2005).

[7] W. V. Liu and F. Wilczek, Phys. Rev. Lett. 90, 047002 (2003).

[8] M. Iskin and C. A. R. Sá de Melo, Phys. Rev. Lett. 97, 100404 (2006).

[9] S.-T. Wu et al., Phys. Rev. B 74, 224504 (2006).

[10] G.-D. Lin et al., Phys. Rev. A 74, 031604(R) (2006).

[11] M. M. Forbes et al., Phys. Rev. Lett. 94, 017001 (2005).

[12] P. F. Bedaque et al., Phys. Rev. Lett. 91, 247002 (2003).

[13] M. M. Parish et al., Nature Phys. 3, 124 (2007).

[14] K. B. Gubbels et al., Phys. Rev. Lett. 97, 210402 (2006).

[15] P. Fulde and R. A. Ferrell, Phys. Rev. 135, A550 (1964); A. I. Larkin and Yu. N. Ovchinnikov, Zh. Eksp. Teor. Fiz. 47, 1136 (1964) [Sov. Phys. JETP 20, 762 (1965)].

[16] T. Köhler et al., Rev. Mod. Phys. 78, 1311 (2006).

[17] The definition of $m$ also leads to the symmetry $(m, r) \mapsto$ $(-m, 1 / r)$, allowing restriction to $m \geq 0$ when varying $r$.

[18] We define the Fermi momentum with respect to average density $n / 2$, so that $k_{F}=\left(3 \pi^{2} n\right)^{1 / 3}$ and $\varepsilon_{F}=k_{F}^{2} / 2 m_{r}$.

[19] This feature survives Nozières-Schmitt-Rink fluctuation corrections, as in the equal mass case [13], but other corrections that include interactions between excess fermions and molecular bosons may modify it.

[20] The existence of a BP-2 phase for $r \ll 1$ was discussed in Ref. [7], but the possibility of phase separation was ignored.

[21] M. M. Parish et al., Phys. Rev. B 71, 064513 (2005).

[22] For zero polarization, while it is true that the ground state remains insensitive to mass ratio (as emphasized in Ref. [9]), the quasiparticle excitation spectrum and its associated DOS are strongly dependent on it. In particular, only one branch of the spectrum has a minimum at nonzero $\mathbf{k}$ when $|\mu / \Delta|<|r-1| / 2 \sqrt{r}$, while both branches have minima at nonzero $\mathbf{k}$ when $|r-1| / 2 \sqrt{r}<$ $\mu / \Delta$ and neither branch possesses them when $\mu / \Delta<$ $-|r-1| / 2 \sqrt{r}$

[23] P. Törmä and P. Zoller, Phys. Rev. Lett. 85, 487 (2000).

[24] M. M. Parish et al., Phys. Rev. Lett. 94, 240402 (2005); J. M. Acton et al., Phys. Rev. A 71, 063606 (2005).

[25] E. Altman et al., Phys. Rev. A 70, 013603 (2004).

[26] P. Noziéres and S. Schmitt-Rink, J. Low Temp. Phys. 59, 195 (1985).

[27] Equivalently, the whole superfluid region will shift to the $h<0$ plane if $1 / r \gtrsim 3.95$. 